\title{
Light flavour hadron production in the ALICE experiment at LHC
}

\author{
Angela Badalì for the AliCE Collaboration
}

INFN, Sezione di Catania, Via S. Sofia 64, I95123, Catania, Italy

\begin{abstract}
Unique among the LHC experiments, ALICE has excellent particle identification capabilities for the measurement of light-flavour hadrons. A large number of hadron species from pions to multi-strange baryons and light nuclei have been measured over a large transverse momentum region. The measurement of the production of these particles is a valuable tool to study the properties of the medium formed in heavy-ion collisions. In particular they give information on the collective phenomena of the fireball, on the parton energy loss in the hot QCD medium and on the hadronization mechanisms such as recombination and statistical hadronization. The measurements in pp and in p-nucleus collisions provide the necessary baseline for heavy-ion data and help to investigate the effects of the ordinary nuclear matter. In this paper some of the main ALICE results on identified light-flavour hadron production in $\mathrm{Pb}-\mathrm{Pb}$ collisions at $\sqrt{s_{\mathrm{NN}}}=2.76 \mathrm{TeV}$ and $\mathrm{p}-\mathrm{Pb}$ collisions at $\sqrt{s_{\mathrm{NN}}}=5.02 \mathrm{TeV}$ will be presented.
\end{abstract}

\section{Introduction}

Heavy-ion collisions at ultra-relativistic energies allow one to study the physics of strongly interacting matter and to characterize the Quark-Gluon Plasma (QGP), a state of deconfined quarks and gluons. The hot and dense matter created in such collisions rapidly expands collectively and cools down, until the (pseudo)-critical temperature is reached and the phase transition (or a crossover at very low $\mu_{B}$ ) to a hadron gas takes place. Hadron yields 
measured in central heavy-ion collisions from AGS up to LHC energies can be described very well within a hadro-chemical equilibrium model $[1,2]$. Then in high-energy interactions hadrons are expected to be produced in approximate chemical and thermal equilibrium. Chemical freeze-out takes place when inelastic collisions stop, while kinetic freeze-out happens after the chemical freeze-out, once also elastic collisions stop.

The production of particles formed only by light quarks $(\mathrm{u}, \mathrm{d}, \mathrm{s})$ is an important tool not only for the understanding of particle production mechanisms in high energy collisions but also to study the collective phenomena characterizing the dynamical evolution of the fireball. In particular, the study of particles with strangeness helps in understanding of the hadrochemistry of the matter created in the collisions since no net strangeness is present in the colliding nuclei. Moreover, high energy heavy-ion collisions offer the opportunity to study light (anti-)nuclei (such as d, ${ }^{3} \mathrm{He},{ }^{4} \mathrm{He}$ ) and hypernuclei (such as ${ }_{\Lambda}^{3} \mathrm{H}$ ) and to test their production mechanisms. Furthermore, production of hadronic resonances (such as $\mathrm{K}^{*}(892)^{0}, \Lambda(1520)$ ) can help in understanding the late hadronic phase.

In the next section, following a brief description of the ALICE detector and the analysis techniques used to identify particles, selected results on light-flavour hadrons and nuclei in $\mathrm{Pb}-\mathrm{Pb}$ collisions at $\sqrt{s_{\mathrm{NN}}}=2.76 \mathrm{TeV}$ and $\mathrm{p}-\mathrm{Pb}$ collisions at $\sqrt{s_{\mathrm{NN}}}=5.02 \mathrm{TeV}$ are presented and discussed.

\section{Particle identification in ALICE and selected results}

A detailed review of the ALICE detector and its particle identification capabilities can be found in $[3,4]$. Charged hadrons in the low to intermediate $p_{\mathrm{T}}$ region (below $5 \mathrm{GeV} / c$ ) are identified using the energy loss $\mathrm{d} E / \mathrm{d} x$ from the Inner Tracking System (ITS) and Time Projection Chamber (TPC) detectors, the time-of-flight measurement from the Time Of Flight (TOF) detector and the Cherenkov light from the High Momentum Particle Identification Detector (HMPID). At higher $p_{\mathrm{T}}$ particles are identified via the $\mathrm{d} E / \mathrm{d} x$ in the relativistic rise range of the Bethe-Bloch curve in the TPC. Also nuclei and anti-nuclei are identified over a wide range of momentum using the combined information of the specific energy loss in the TPC and the velocity measured by the TOF. More information can be found in [5], which reports the recent $\mathrm{CPT}$ invariance test in the systems bound by nuclear forces obtained by ALICE measuring the difference between the ratios of the mass and the charge of $\mathrm{d}$ and $\overline{\mathrm{d}},{ }^{3} \mathrm{He}$ and ${ }^{3} \overline{\mathrm{He}}$ nuclei. Strange 
hadrons are measured through the reconstruction of their weak decay into charged particles. $\mathrm{K}_{\mathrm{S}}^{0}, \Lambda, \bar{\Lambda},{ }_{\Lambda}^{3} \mathrm{H}, \frac{3}{\Lambda} \overline{\mathrm{H}}$ candidates are found by combining two charged tracks in a $\mathrm{V}$-shaped decay pattern while for multi-strange baryons (charged $\Xi$ and $\Omega$ ) a selected $\Lambda$ candidate is further combined with a third track. Candidates are required to satisfy topological and kinematical restrictions. In addition, each of the daughter tracks is checked for compatibility with the pion, kaon, proton or ${ }^{3} \mathrm{He}$ hypotheses using their energy loss in the TPC. A description of the identification of hadronic resonances (as $\mathrm{K}^{*}$ and $\phi$ ) can be found in [6].

The transverse momentum $\left(p_{\mathrm{T}}\right)$ spectra of identified hadrons $\pi^{ \pm}, \mathrm{K}^{ \pm}$and $\mathrm{p}(\overline{\mathrm{p}})$ in $\mathrm{Pb}-\mathrm{Pb}$ collisions show a clear evolution with the centrality, with an almost exponential behaviour at high $p_{\mathrm{T}}$. The spectrum hardens at high $p_{\mathrm{T}}$ and flattens at low $p_{\mathrm{T}}$ as a function of increasing centrality. The $\left\langle\beta_{T}\right\rangle$ parameter extracted from fits to the blast-wave parameterization indicates a radial flow of about $10 \%$ higher than at RHIC at $\sqrt{s_{\mathrm{NN}}}=200 \mathrm{GeV}$ in central collisions [7]. The $p_{\mathrm{T}}$ spectra are succesfully described [7] by hydrodynamic models (VISH2+1 [8], HKM [9], Kraków [10], EPOS [11]). Comparison with prediction from hydrodynamic calculations, based on the same models used for $\pi, \mathrm{K}$ and $\mathrm{p}$ have also been carried out for the heavier multistrange baryons [12]. For the most central events (0-10\%), all the available models describe quite well the shape of the $\Xi$ spectrum in the $p_{\mathrm{T}}$ range up to $3 \mathrm{GeV} / c$, although only the Kraków model correctly reproduces the yield. The description is less successful with the $\Omega$. Considering that for the hydrodynamic description the shape of the transverse momentum distribution is mainly determined by particle mass, it is interesting to note that for $p_{\mathrm{T}}<3 \mathrm{GeV} / c$ the $\phi$-mesons and protons $p_{\mathrm{T}}$ spectra have a similar shape [6].

The $p_{\mathrm{T}}$ spectra of $\pi^{ \pm}, \mathrm{K}^{ \pm}, \mathrm{p}(\overline{\mathrm{p}})$ and $\Lambda(\bar{\Lambda})$ have been measured in $\mathrm{p}-\mathrm{Pb}$ collisions in different VZERO multiplicity classes [13]. For $p_{\mathrm{T}}<2 \mathrm{GeV} / c$ the spectra become harder as the multiplicity increases and the effect is stronger for heavier particles. This pattern is similar to the one observed in $\mathrm{Pb}-\mathrm{Pb}$ collisions, where it is attributed to the hydrodynamical evolution of the medium and indeed also $p_{\mathrm{T}}$ spectra measured in highmultiplicity $\mathrm{p}-\mathrm{Pb}$ collisions are better described by hydrodynamical models (EPOS [11], Kraków [10]) than QCD inspired models (DPMJET [14]).

Ultrarelativistic heavy-ion collisions offer also the opportunity to study light anti-(hyper) nuclei. Even if the measurement is challenging as the production probability decreases with increasing mass, the data collected with the ALICE detector during the first LHC run phase allow for the identification and measurement of d, $\overline{\mathrm{d}},{ }^{3} \mathrm{He},{ }^{3} \overline{\mathrm{He}}[15],{ }^{4} \overline{\mathrm{He}}$ and (anti-)hypertriton ${ }_{\Lambda}^{3} \mathrm{H}[16]$ in $\mathrm{Pb}-\mathrm{Pb}$ collisions at $\sqrt{s_{\mathrm{NN}}}=2.76 \mathrm{TeV}$. As expected in a hydrody- 
namic description of the fireball as a radially expanding source, a hardening of the spectra with increasing centrality is observed [15]. The decrease with the increasing mass of the nuclei of the $p_{\mathrm{T}}$-integrated yield of $\mathrm{p}, \mathrm{d},{ }^{3} \mathrm{He}$ and ${ }^{4} \overline{\mathrm{He}}$ in $0-20 \%$ centrality nicely follows the exponential fall predicted by the thermal model, with a penalty factor of about 300 for each added baryon. The (anti-)hypertriton $\left(\frac{3}{\Lambda} \overline{\mathrm{H}}\right){ }_{\Lambda}^{3} \mathrm{H}$ is the lightest observed hypernucleus and is formed by a (anti-)proton, a (anti-)neutron and a (anti-) $\Lambda$. The statistics reached for the hypertriton (particle + anti-particle) has permitted to estimate the yield in four $c t$ bins. From a fit of these measurements a lifetime of $181_{-39}^{+54}$ (stat.) \pm 33 (syst.) ps for the ${ }_{\Lambda}^{3} \mathrm{H}$ was determined which is compatible within $1 \sigma$ to the world average value [16].
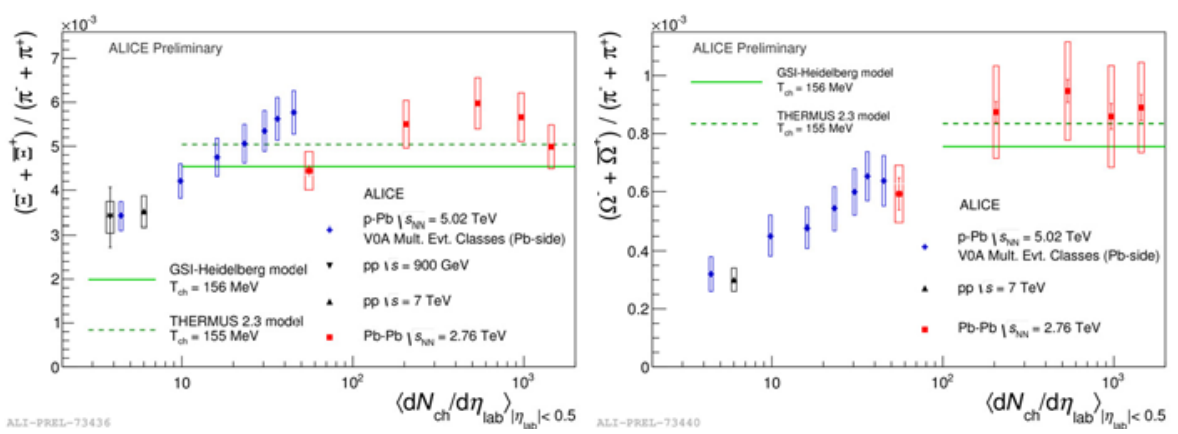

Figure 1: Multi-strange hyperon-to-pion ratios as a function of multiplicity (centrality) for $\mathrm{pp}$ (minimum-bias), $\mathrm{p}-\mathrm{Pb}$ and $\mathrm{Pb}-\mathrm{Pb}$ collisions shown together with predictions from statistical hadronizations models.

Since the production rate of charged particles does not scale linearly with $\left\langle N_{\text {part }}\right\rangle$ [17] a way to factor out the enhancement due to strangeness content from the overall increase in charged-particle production is to compute the ratio of strange particle to pions. A clear increase of strangeness production is observed from minimum-bias pp to central $\mathrm{Pb}-\mathrm{Pb}$ collisions [12]. In Fig. 1 the $\frac{\Xi^{-}+\bar{\Xi}^{+}}{\pi^{-}+\pi^{+}}$and $\frac{\Omega^{-}+\bar{\Omega}^{+}}{\pi^{-}+\pi^{+}}$ratios for the three collisions systems are reported as a function of the averaged charged multiplicity. The multi-strange baryon production relative to pions increases by up to a factor of about 3 when going from minimum-bias pp to central $\mathrm{Pb}-\mathrm{Pb}$. It saturates in the most central $\mathrm{Pb}-\mathrm{Pb}$ collisions at values predicted by statistical hadronization models. In $\mathrm{p}-\mathrm{Pb}$ collisions these ratios increase with multiplicity, with $\Xi / \pi$ reaching the saturation level observed in central $\mathrm{Pb}-\mathrm{Pb}$ collisions and $\Omega / \pi$ being compatible with the value for peripheral $\mathrm{Pb}-\mathrm{Pb}$ collisions. A similar trend is observed for the $\mathrm{d} / \mathrm{p}$ ratio (not shown). It increases with multiplicity in 
$\mathrm{p}-\mathrm{Pb}$ collisions bridging the $\mathrm{pp}$ and $\mathrm{Pb}-\mathrm{Pb}$ values. However, within errors, no significant centrality dependence is observed in $\mathrm{Pb}-\mathrm{Pb}$ collisions.

In high-energy interactions hadrons are expected to be produced in approximate chemical and thermal equilibrium. A grand-canonical model is usually employed to describe the observed yields of each particle species in nucleus-nucleus collisions. The mid-rapidity $p_{\mathrm{T}}$-integrated yield of light flavour hadrons (including nuclei) measured by ALICE in central $\mathrm{Pb}-\mathrm{Pb}$ collisions at $\sqrt{s_{\mathrm{NN}}}=2.76 \mathrm{TeV}$ have been fitted to three statistical hadronization models (THERMUS 2.3 [18], GSI-Hidelberg [19] and SHARE [20]) with the baryochemical potential set to $1 \mathrm{MeV}$. The yields are described over 7 orders of magnitude with a common $\mathrm{T}_{\text {chemical }}=156 \pm 2 \mathrm{MeV}$, while fits to RHIC data resukted in a slightlt higher temperature $(\sim 164 \mathrm{MeV})$. However the models largely overestimate the proton and anti-proton yields. Some of the suggested interpretations of this 'anomaly' are: baryon-antibaryon annhihilation after chemical freeze-out, non-equilibrium statistical hadronization and effects due to pre-hadronic flavour-dependent bound states above the QCD transition temperature (see ref. [21] for details). Probably due to the importance of rescattering effects in the late hadronic phase the $\mathrm{K}^{*}(892)^{0}$ resonance yield, excluded from the fit, is also overestimated.

\section{Summary and conclusions}

The ALICE experiment has performed a comprehensive study of the light flavour hadrons and light nuclei, in a wide range of $p_{\mathrm{T}}$ for events collected in $\mathrm{pp}, \mathrm{p}-\mathrm{Pb}$ and $\mathrm{Pb}-\mathrm{Pb}$ collisions during the first $\mathrm{LHC}$ run phase. Bulk particle production in $\mathrm{p}-\mathrm{Pb}$ shows a clear evolution with multiplicity similar to the pattern observed in $\mathrm{Pb}-\mathrm{Pb}$ collisions, where it is considered a signature of collectivity. However we should be aware that also non-collective effects can mimic flow-like patterns [13]. Particle production changes with increasing the colliding system size. In particular, the relative suppression of baryon and $\mathrm{K}^{*}(892)^{0}$ and the relative enhancement of strangeness and deuteron production are observed. In central $\mathrm{Pb}-\mathrm{Pb}$ collisions $p_{\mathrm{T}}$-integrated yields of light flavour particles fit the predictions from statistical hadronization with a chemical freeze-out temperature around $156 \mathrm{MeV}$. Production of light (hyper) nuclei is found to be in agreement with thermal model expectation, despite their low binding energies. Their inclusion in chemical equilibrium fits, which might seem questionable, can be understood if one consideres that the hadronic interactions do not change the entropy per baryon and the statistical hadronization [1] and coalescence [22] approach yield compatible results [15]. 


\section{References}

[1] A. Andronic et al., Nucl. Phys. A 772 (2006) 167; J. Stachel et al, J. Phys. Conf. Ser. 509 (2014) 012019, arXiv 1311.4662.

[2] F. Becattini et al., Phys. Rev. C 90 (2014) 054907.

[3] K. Aamodt et al. (ALICE Coll.), J. Instrum. 3 (2008) S08002.

[4] B. Abelev et al. (ALICE Coll.), Int. J. Mod. Phys. A 29 (2014) 1430044.

[5] B. Abelev et al. (ALICE Coll.), arXiv:1506.08951; B. Abelev et al. (ALICE Coll.), Nat. Phys. Lett. NPHYS34432 (2015).

[6] B. Abelev et al. (ALICE. Coll.), Phys. Rev. C 91 (2015) 024609.

[7] B. Abelev et al. (ALICE. Coll.), Phys. Rev. C 88 (2013) 044910.

[8] C. Shen et al., Phys. Rev. C 84 (2011) 044903.

[9] Y. Karpenko and Y. Senyukov, J. Phys. G 38 (2011) 124059.

[10] P. Bozek, Phys. Rev. C 85 (2012) 034901.

[11] K. Werner, Phys. Rev. C 85 (2012) 064907.

[12] B. Abelev et al. (ALICE. Coll.), Phys. Lett. B 728 (2014) 216.

[13] B. Abelev et al. (ALICE. Coll.), Phys. Lett. B 728 (2014) 25.

[14] S. Roesler et al., arXiv:hep-ph 0012252.

[15] J. Adam et al. (ALICE Coll.), arXiv: 1506.08951.

[16] J. Adam et al. (ALICE Coll.), arXiv: 1506.08453.

[17] K. Aamodt et al. (ALICE Coll.), Phys. Rev. Lett. 106 (2011) 032301.

[18] S. Wheaton et al., Comp. Phys. Commun. 180 (2009) 8.

[19] A. Andronic et al., Phys. Lett. B 673 (2009)14.

[20] M. Petran et al., Comp. Phys. Comm. 185 (2014) 2056.

[21] M. Floris, Nucl. Phys. A 931 (2014) 103.

[22] P. Siemens and J.I. Kapusta, Phys. Rev. Lett. 43 (1979) 1486. 